\title{
The Conformation of Amylose in Solution. I.*
}

\author{
Akira Hayashi, Kyosuke KinOShita, and Yasuo MiYaKe \\ Department of Chemistry, Ritsumeikan University, \\ 28 Tojiinkita-machi, Kita-ku, Kyoto 603, Japan
}

(Received September 3, 1980)

\begin{abstract}
The neutralization process of an alkalline solution of amylose was investigated by fluorescence depolarization and optical rotation to find the conformation of this polysaccharide in aqueous solution. The measurements showed that amylose existed in different conformations in alkaline and neutral solutions. The transition between these conformations appeared as a minimum in the $1 / p$ and $[\alpha]_{405} v s$. $\mathrm{pH}$ curves at $\mathrm{pH} 12.5$. Here $p$ is the degree of polarization and $[\alpha]_{405}$ is the specific optical rotation at $405 \mathrm{~nm}$ wavelength. In an alkalline solution, the reduced viscosity showed behavior characteristic of polyelectrolytes and the conformation of the polymer seemed to be a negatively charged expanded coil. But in a neutral solution, the polymer seemed to take on an ordered conformation. This ordered conformation disappeared when KSCN was added to the solution, implying that it was maintained by hydrogen bonds. This conformation also disappeared in two steps, at about 35 and $60^{\circ} \mathrm{C}$ on heating. One step, at the higher temperature, consists in the transition from helix to coil. This temperature agrees with the "gelatinization temperature" of amylose or starch. The other step, at the lower temperature, is a secondary change in the helical structure. The transition from coil to helix occurred in a $\mathrm{pH}$ range from 13 to 12 . The salt produced by neutralization of the alkalline amylose solution seemed to allow the amylose molecules to shrink to compact coils before winding helical turns.

KEY WORDS Amylose / Conformation / Fluorescence Depolarization /

Helix-Coil Transition / Gelationization Temperature /
\end{abstract}

Models such as the random coil, ${ }^{1}$ interrupted helix, ${ }^{2}$ and the wormlike helix ${ }^{3}$ have been proposed to explain the conformation of amylose in aqueous solution, but we do not as yet know what model among these is the best. ${ }^{4}$ In this work, we examined the $\mathrm{pH}$ and temperature dependence of the fluorescence depolarization and optical rotation of aqueous amylose solutions in the presence and absence of KSCN, hoping to obtain information which could be used to establish the correct conformation of amylose in solution.

The $\mathrm{pH}$ dependence of the conformation of amylose was investigated by $\mathrm{Rao}^{3}$ and also by Greenwood, ${ }^{1}$ but the conclusions drawn by these authors are at variance. Rao proposed an expanded coil, a compact coil, and a wormlike helix for this polymer in alkalline, transient, and neutral so-

* Presented at the Annual Meeting of the Agricultural Chemical Society of Japan, Fukuoka, Japan, April 1-4, 1980. lutions, respectively. Greenwood, however, suggested a random coil, a helix, and a random coil in these solutions. We consider that such a difference is due merely to the difference in the interpretation of molecular parameter values determined from viscometric, ultracentrifugal, and light-scattering measurements and thus not essential.

\section{EXPERIMENTAL}

\section{Materials}

An AVEBE amylose-V was used as the sample for the present study. All measurements were made at a single amylose concentration of $0.01 \mathrm{~g} \mathrm{dl}^{-1}$ unless otherwise stated.

Uranine was used as the fluorescent dye and dissolved in a given solution at a concentration of $10^{-6} \mathrm{M}$. Spectrophotometric analysis showed that uranine formed no complex with amylose even in a neutral solution.

Other chemicals used were of reagent grade. 


\section{Methods}

Fluorescence polarization measurements were made using a Hitachi Fluorescence Spectrophotometer 204 provided with a polarizer and analyzer. The technical details are same as those described in our previous papers on agarose. ${ }^{6-7}$

The specific optical rotation was measured by a Union Automatic Digital Polarimeter PM-101, which was capable of detecting rotation down to $10^{-4}$ degree. This measurement was made at a $405 \mathrm{~nm}$ wavelength.

The relative viscosity was measured by a Ubbelohde-type viscometer at $25^{\circ} \mathrm{C}$.

A Hitachi 100-50 Spectrophotometer was used to measure the difference spectrum for $p$-nitrophenol (PNP) between an aqueous solution containing $0.01 \mathrm{~g} \mathrm{dl}^{-1}$ amylose and $5 \times 10^{-5} \mathrm{M} \mathrm{PNP}$ and a solution containing only $5 \times 10^{-5} \mathrm{M}$ PNP.

An acid titration of an alkalline amylose solution was carried out in the following way. An aliquot of $0.1 \mathrm{~g} \mathrm{dl}^{-1}$ amylose dissolved in $1 \mathrm{~N} \mathrm{KOH}$ was diluted by appropriate volumes of $0.2 \mathrm{~N} \mathrm{HCl}$ and water to yield an $0.01 \mathrm{~g} \mathrm{dl}^{-1}$ amylose solution. Then $0.2 \mathrm{~N} \mathrm{HCl}$ was added stepwise to the solution and the $\mathrm{pH}$ was measured after each dilution. The $\mathrm{pH}$ meter was calibrated with a standard buffer of $\mathrm{pH} 9$ and $0.1 \mathrm{~N} \mathrm{KOH}$.

\section{RESULTS}

Figure 1 shows $1 / p$ and $[\alpha]_{405}$ at $25^{\circ} \mathrm{C}$ as a function of $\mathrm{pH}$. Here $p$ is the degree of polarization and $[\alpha]_{405}$ is the specific optical rotation at $405 \mathrm{~nm}$ wavelength. It is seen that both $1 / p$ and $[\alpha]_{405}$ exhibit a sharp minimum at a $\mathrm{pH}$ of about $12.5 \cdot \mathrm{Rao}^{3}$

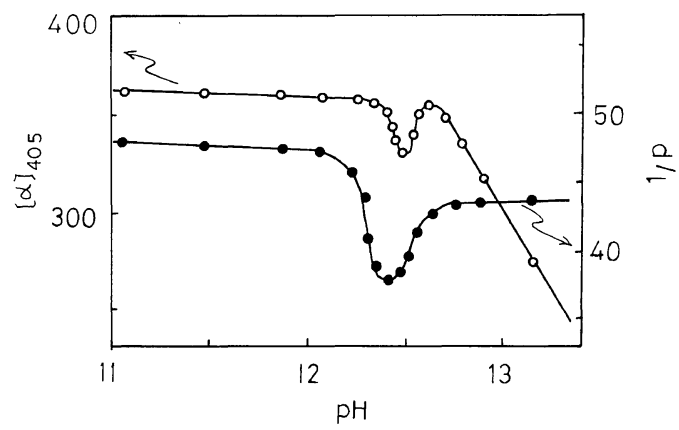

Figure 1. Dependence on $\mathrm{pH}$ of the specific optical rotation at $405 \mathrm{~nm}(-\bigcirc-)$ and fluorescence depolarization (--) of amylose solution.

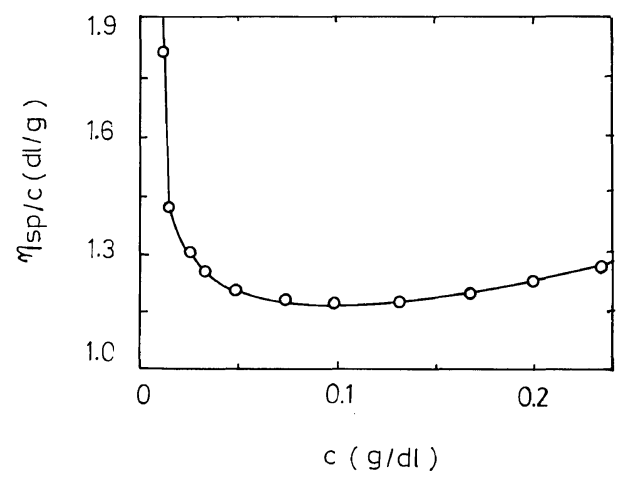

Figure 2. Dependence of the reduced viscosity on concentration in $1 \mathrm{~N} \mathrm{KOH}$.

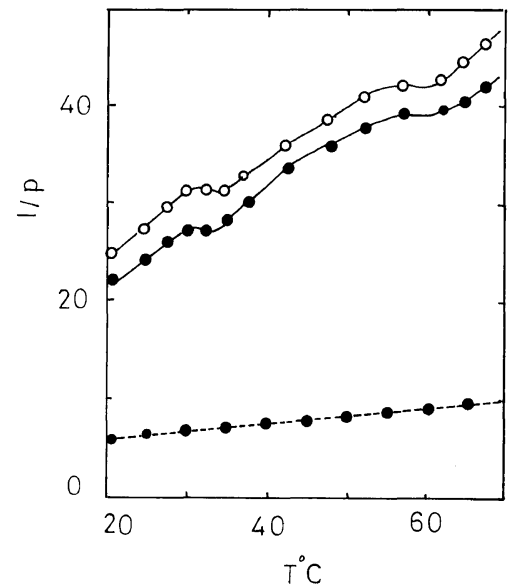

Figure 3. Dependence of the fluorescence depolarization on temperature for amylose in neutral solution (pH 8.3) containing no $\mathrm{KSCN}(-\mathrm{O}-), 0.2 \mathrm{~N} \mathrm{KSCN}$ (一-), and $2 N \mathrm{KSCN}(-----)$.

observed, in his study on amylose, a minimum of $[\eta]$ (intrinsic viscosity) at $\mathrm{pH} 12$, but no minimum of $[\alpha]$ over the entire range of $\mathrm{pH}$ studied.

Figure 2 illustrates the concentration dependence of reduced viscosity $\eta_{\text {sp }} / c$ in $1 N \mathrm{KOH}$ at $25^{\circ} \mathrm{C}$. The curve shows a sharp rise at low polymer concentration as is usually observed with polyelectrolytes. This behavior suggests that amylose in alkalline solution $(\mathrm{pH}>13)$ is electrically charged.

The three curves in Figure 3 show variation in $1 / p$ observed when the temperature was raised in neutral solutions ( $\mathrm{pH} 8.3$ ) containing no, $0.2 \mathrm{~N}$, and $2 \mathrm{~N}$ $\mathrm{KSCN}$. The two upper curves exhibit dents at about 35 and $60^{\circ} \mathrm{C}$, but no dent is observed in the lowest curve. These data suggest that the conformation of 
The Conformation of Amylose in Solution. I.

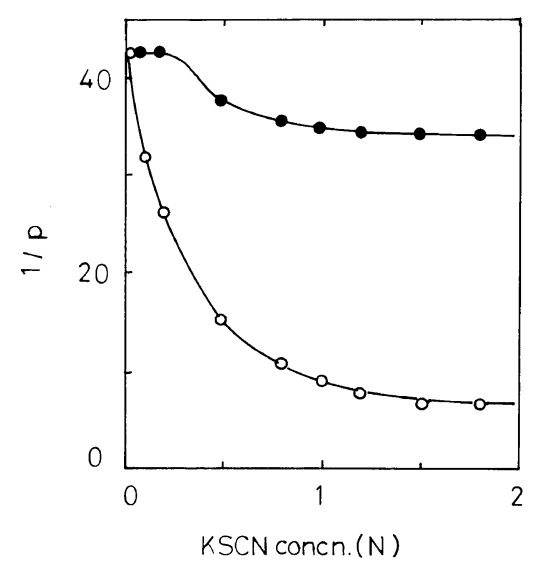

Figure 4. Dependence of the fluorescence depolarization on concentration of $\mathrm{KSCN}(-\mathrm{O}-$ ) and its corrected curve (- -) at $\mathrm{pH} 8.3$.

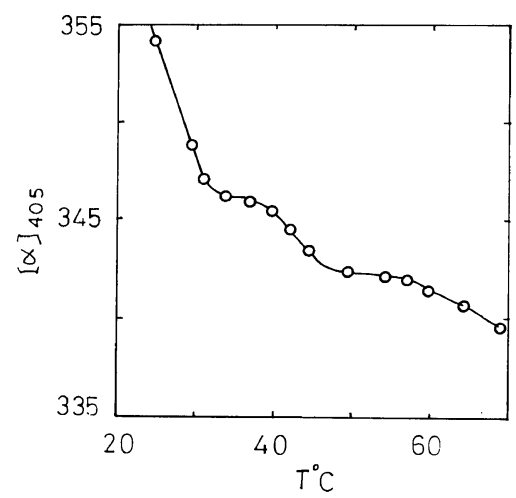

Figure 5. Dependence of the specific optical rotation on temperature for amylose in neutral solution ( $\mathrm{pH} 8.3$ ).

amylose in a neutral solution $(\mathrm{pH}<12)$ has something to do with hydrogen bonding, since $\mathrm{KSCN}$ is a typical reagent for hydrogen bond breaking.

The lower curve in Figure 4 indicates $1 / p$ at $25^{\circ} \mathrm{C}$ as a function of $\mathrm{KSCN}$ added to the solution of $\mathrm{pH}$ 8.3. This curve should be corrected for the decrease in the lifetime of the fluorescent dye that is caused by the addition of $\mathrm{KSCN}$ in order to see the mobility of the polymer segment. This correction was made by measuring $1 / p$ of the blank solution containing only the dye. The upper curve shows the result obtained following this correction. This result indicates that the amylose molecule in a neutral solution undergoes "denaturation" and that the denaturation is completed before the concentration

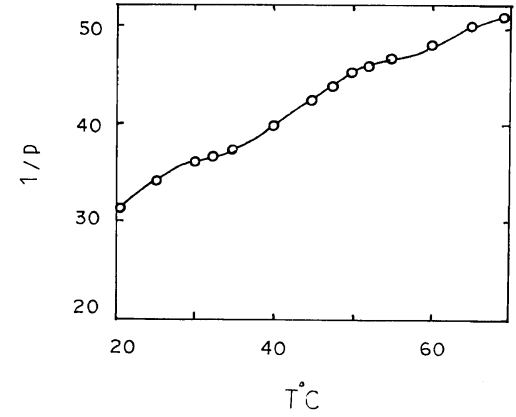

Figure 6. Dependence of the fluorescence depolarization on temperature for amylose in the solution of $\mathrm{pH}$ 12.5.

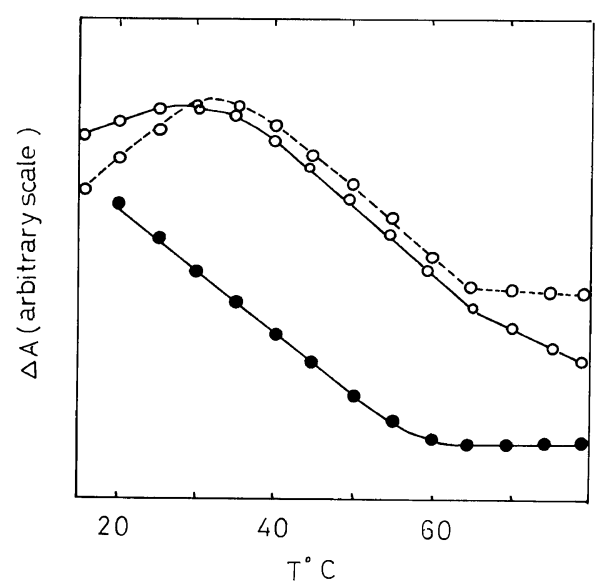

Figure 7. Dependence on temperature of the differential absorbance at $400 \mathrm{~nm}(-\bigcirc-)$ and $310 \mathrm{~nm}\left(--\bigcirc_{---)}\right.$ for PNP-amylose complex and $\mathrm{PNP}-\alpha$-cyclodextrin complex (- - ) at $\mathrm{pH} 8.3$.

of $\mathrm{KSCN}$ reaches $2 N$.

Figure 5 shows $[\alpha]_{405}$ for amylose in a neutral solution ( $\mathrm{pH} 8.3)$ as a function of temperature. It is worth noting that the curve has weak shoulders in those temperature regions where $1 / p$ display dents.

The data shown in Figure 6 is the temperature dependence of $1 / p$ at $\mathrm{pH}$ 12.5. The curve still exhibits dents at the same temperatures as in the neutral solution, though less distinctly. These dents disappeared in alkalline solutions.

The amylose-PNP complex could hardly be observed spectrophotometrically in an alkalline solution. However, the solution of $\mathrm{pH} 8.3$ containing $0.01 \mathrm{~g} \mathrm{dl}^{-1}$ amylose and $5 \times 10^{-5} \mathrm{M}$ PNP was slightly turbid at room temperature, but became 
transparent when heated above about $60^{\circ} \mathrm{C}$. We examined what happens to the difference spectrum between this solution and the blank solution when the temperature exceeds room temperature. The upper two curves in Figure 7 illustrate observed differences $(\Delta A)$ in absorbance at 400 and $310 \mathrm{~nm}$ as a function of temperature. These wavelengths correspond to the absorption bands of $p$ nitrophenolate and nondissociated $p$-nitrophenol. Either curve exhibits a maximum at about $35^{\circ} \mathrm{C}$ and a dent at $65^{\circ} \mathrm{C}$. It should be noted that the higher temperature is close to the temperature at which the neutral solution of amylose containing PNP becomes transparent.

The bottom curve in Figure 7 shows $\Delta A$ at $400 \mathrm{~nm}$ as a function of temperature obtained when the amylose was replaced by $\alpha$-cyclodextrin. This curve has a breaking point at $60^{\circ} \mathrm{C}$, but exhibits no maximum. The solution was transparent throughout the entire temperature range examined.

\section{DISCUSSION}

Studies on agarose and other polymers indicate that $1 / p$ is usually larger when the polymer takes on a random coil conformation than when it has a certain ordered conformation such as helix. ${ }^{5}$ This difference may be interpreted in terms of the microBrownian motion of the polymer segment onto which the fluorescent dye is adsorbed. Thus, the fact illustrated in Figure 1 that $1 / p$ of amylose is lower in an alkalline solution $(\mathrm{pH}>13)$ than in a neutral solution $(\mathrm{pH}<12)$ might be considered evidence that amylose assumes an ordered conformation in an alkalline solution. However, this is not likely to be correct, since amylose is much more soluble in an alkalline solution than in a neutral solution and its viscosity is substantially higher in the former than in the latter solvent.

The polyelectrolyte behavior of reduced viscosity in Figure 2 indicates that amylose becomes a polyanion in alkalline solution. Electrostatic repulsion does not allow the negatively charged uranine molecule to adsorb on such a negatively charged amylose. Thus, the lower $1 / p$ values in an alkalline solution may not be due to an ordered conformation of amylose but to the rotational motion of free uranine molecules.

According to Kitamura, et al., ${ }^{8}$ when uranine is chemically combined with amylose, $1 / p$ of the dye increases as the $\mathrm{pH}$ is increased in an alkalline solution. This suggests that the amylose conformation is less ordered in an alkalline solution than in a neutral solution, although our $1 / p$ data can not distinguish this difference.

In summary, we conclude that the amylose molecule assumes a negatively charged expanded coil in an alkalline solution and an ordered conformation in a neutral solution.

The dents observed at about 35 and $60^{\circ} \mathrm{C}$ in the $1 / p$ vs. temperature curves for amylose in neutral solution (Figure 3) suggest that changes in the conformation of amylose take place at these temperatures. This suggestion is also supported by the shoulders in the $[\alpha]_{405}$ vs. temperature curve in Figure 5. The fact that the dents in the $1 / p v s$. temperature curves disappear completely on adding $2 N \mathrm{KSCN}$ indicates that these changes may be associated with the formation of hydrogen bonds.

We believe that the dent at the higher temperature $60^{\circ} \mathrm{C}$ is an indication of the conformational change from helix to random coil. It is interesting to note that this temperature agrees with the "gelatinization temperature" of amylose or starch, at which amylose or starch granules suspended in water begin to swell on heating. No idea can be offered for the dent at the lower temperature, but it may have something to do with a secondary change in the helix formed at $60^{\circ} \mathrm{C}$.

It is well known that amylose forms a helix called $\mathrm{V}$-amylose in the presence of a guest molecule such as PNP. ${ }^{9}$ The fact that $\Delta A v$ s. temperature curves for amylose-PNP complex (Figure 7) exhibit a maximum and a bent at temperature close to the dents in the $1 / p v s$. temperature curve for amylose (Figure 3) suggests that the same changes occur in the amylose helix at these temperatures when the solution is heated. We consider that the change at the higher temperature is the disappearance of the complex and the helix-coil transition, judging from a comparison with the $\Delta A v s$. temperature curve for the $\alpha$-cyclodextrin-PNP complex (Figure 7). It is interesting that the helix-coil transition point coincides with the complex formation point.

The minimum in $1 / p$ at $\mathrm{pH} 12.5$ is lower than $1 / p$ in a neutral solution (Figure 1). This fact indicates that amylose takes on a less mobile molecular shape at this $\mathrm{pH}$ than the helical conformation in neutral solution. The $[\alpha]_{405}$ value at $\mathrm{pH} 12.5$ is between 
those of the helix in neutral solution and the expanded coil in alakalline solution, although this $\mathrm{pH}$ is within the range of discharged amylose when judged from the form of the $[\alpha]_{405} v s$. $\mathrm{pH}$ curve in Figure 1 . The indistinct dents in the $1 / p v s$. temperature curve at $\mathrm{pH} 12.5$ (Figure 6) indicate that the helix formation is still incomplete at this $\mathrm{pH}$. Summarizing these features, we conclude that the molecular shape of amylose at $\mathrm{pH} 12.5$ at room temperature is a less-swollen compact coil. At this $\mathrm{pH}$, the charged expanded coil loses its negative charges due to $\mathrm{KCl}$ produced by neutralization before it winds complete helical turns and shrinks to a compact coil.

These considerations support Rao's view ${ }^{3}$ on the neutralization of alkalline amylose solution, though not always the details. The most important difference is that the structure of amylose in a neutral solution is a secondary product from the helical segments.

Acknowledgment. The authors wish to express their appreciation to Professor Takashi Kuge of Kyoto Prefectural University for his continued ad- vice and helpful discussion.

\section{REFERENCES}

1. W. Banks and C. T. Greenwood, Stärke, 23, 300 (1971).

2. J. Szejtli, M. Richter, and S. Augustat, Biopolymers, 5, 5 (1967).

3. V. S. R. Rao and J. F. Foster, Biopolymers, 1, 527 (1963).

4. J. Nikuni (Edited), "Denpun Kagaku (Starch Science) Handbook," Asakura, Tokyo, 1977; W. Banks and C. T. Greenwood, "Starch and its Components," Edinburgh University Press, Edinburgh, 1975.

5. A. Hayashi, K. Kinoshita, and M. Kuwano, Polym. J., 9, 219 (1977).

6. A. Hayashi, K. Kinoshita, M. Kuwano, and A. Nose, Polym. J., 10, 485 (1978).

7. A. Hayashi, K. Kinoshita, and S. Yasueda, Polym. J., 12, 447 (1980).

8. S. Kitamura, H. Yunokawa, and T. Kuge, presented at the Annual Meeting of the Agricicultural Chemical Society of Japan, Fukuoka, April 1-4, 1980.

9. P. R. Sundararajan and V. S. R. Rao, Biopolymers, 8, 313 (1969). 